\title{
Trastorno del espectro del autismo. Implicaciones en la práctica clínica de una conceptualización basada en el déficit.
}

Autism spectrum disorder. Clinical implications of a deficit-based conceptualization.

\author{
Carlos M. Jordán Cristóbal a . \\ ${ }^{a}$ Psicólogo Clínico. Equipo Oeste. Gabinete de Psicología Clínica. Boadilla del Monte, Madrid, España.
}

Correspondencia: Carlos M. Jordán Cristóbal (carlos@equipooeste.com)

Recibido: 10/03/2015; aceptado: 18/07/2015

\begin{abstract}
RESUMEN: Con la aparición del DSM-5 se consolida una visión del autismo en torno a los conceptos de espectro y discapacidad. De esta visión se derivan intervenciones que tratan de compensar los déficits encontrados mediante la educación adaptada y la modificación de conducta, un enfoque heredado de la discapacidad intelectual. En este trabajo se analiza el devenir del concepto de autismo infantil (desde sus orígenes ligados a la esquizofrenia y las psicosis infantiles hasta la actual consolidación de la idea de espectro autista) como punto de partida para entender de qué forma su desvinculación de la psicopatología clásica está afectando a la comprensión de estos cuadros y, como consecuencia, a las intervenciones que se proponen. Además, mediante una pequeña incursión en la clínica con dos viñetas, intentamos rescatar una visión del autismo como algo más que un déficit, como una construcción positiva, una defensa contra algún tipo de sufrimiento, sin que por ello haya que rechazar las intervenciones compensatorias.

PALABRAS CLAVE: trastorno autístico, Trastornos Generalizados del Desarrollo Infantil, trastornos psicóticos, Síndrome de Asperger.
\end{abstract}

ABSTRACT: With the appearance of DSM-5 a vision of autism based on the concepts of spectrum and disability has been consolidated. From this vision emerge a kind of interventions that try to compensate deficits by means of educational adaptations and behaviour therapy, an approach inherited from that applied to intellectual disability. In this work we discuss the development of the concept of infantile autism (from his origins tied to schizophrenia and infantile psychoses up to the current consolidation of the idea of autistic spectrum) as a starting point to understand in which way the detachment from classic psychopathology is changing our comprehension of these clinical pictures and, as a consequence, the way we trait them. By means of a small incursion into the clinic with two vignettes, we also intend to take up the idea of autism as something more than a deficit, a positive construction, a harsh defence against some sort of suffering, without thereby having to abandon compensatory interventions.

KEY WORDS: Autistic disorder, Pervasive Child Development Disorders, psychotic disorders, Asperger Syndrome.

\section{Introducción}

Este trabajo tiene como origen tres circunstancias que nos condujeron a reflexionar sobre la relación que existe entre el tratamiento del autismo y los postulados teóricos que sustentan su diagnóstico. Dichas circunstancias fueron: el trabajo individual con pacientes graves en un Centro de Salud Mental de la Comunidad de Madrid, la publicación del DSM-5 (1) y un pequeño trabajo de revisión de la historia del concepto de autismo infantil llevado a cabo por motivos clínicos. El resultado, 
sin ser propiamente ni un estudio de casos ni una revisión sistemática, plantea -a nuestro entender- un asunto delicado: las teorías implícitas en los diagnósticos que utilizamos dejan fuera del tratamiento lo que dejan fuera de su conceptualización.

Partiremos de que la actual conceptualización del autismo - centrada en la idea de déficit- empuja a una forma de intervención educativa y de control de síntomas basada en principios de aprendizaje y modificación de conducta (2). Si hemos rastreado el camino que nos ha llevado a la situación actual en este terreno, a conocer sus orígenes teóricos, no es porque pretendamos invalidar dichas intervenciones -pues en gran medida parecen necesarias-, sino para señalar que, probablemente, estamos dejando fuera del foco cuestiones vitales para poder entender a estos pacientes. Al mismo tiempo, nos gustaría recordar que otras formas de pensar el autismo pueden apoyar la necesidad de intervenciones que van más allá del aprendizaje casi mecánico de determinadas habilidades.

\section{$D S M-5$}

Aunque algunas polémicas que suscitaron los adelantos del DSM-5 (1) se han zanjado dejando las cosas como estaban (p. ej. los trastornos de la personalidad), no ha sido así en el apartado infantil, dominado desde el título por un sustancial cambio en forma de postulado etiológico, al sustituir los "Trastornos de inicio en la infancia, la niñez o la adolescencia" por los "Trastornos del desarrollo neurológico". Parece que, acorde con tiempos en los que están cayendo muchas máscaras, el manual renuncia a la simulada posición "ateórica y descriptiva", estandarte de la clasificación americana desde hace décadas (3). En este punto, se puede considerar más honrado que sus antecesores, pues la teoría no es escamoteada, sino puesta por delante.

En nuestro caso, no somos del todo partícipes de la indignación generada por el nuevo DSM, indignación que ha alcanzado, incluso, a ilustres miembros de la APA que participaron en la elaboración de la versión anterior (4). Creemos que, en algunos aspectos, el polémico texto no hace sino intentar revestir de rigor académico-científico lo que en la práctica clínica es casi una norma. Por nombrar dos de las controversias suscitadas por el manual y más cercanas a la consulta diaria: 1) El Síndrome de Psicosis Atenuado, enclavado en el "Espectro de la Esquizofrenia y otros Trastornos Psicóticos", podría servir para encajar prácticas que empiezan a popularizarse, como el abordaje preventivo de las psicosis (5), sin que se tenga muy en cuenta que ya disponemos de literatura que nos alerta "del peligro que puede suponer ser población de riesgo" ("The risks of being at risk", en el original (6)), o el que pueden conllevar las intervenciones precoces y muy precoces en este tipo de cuadros (7). Otro cambio en el DSM-5 que puede reflejar prácticas habituales es la nueva y complicada forma 
de diferenciar entre duelo y depresión, que supuestamente abriría las puertas a la prescripción de antidepresivos durante el duelo, algo que, en realidad, no era una excepción antes de su publicación, al menos en atención primaria (8).

Por lo tanto, gran parte del revuelo se debería a ver negro sobre blanco usos y costumbres bastante extendidas o novedades que son aceptadas de facto sin que se preste demasiada atención a la literatura crítica. En esta misma situación se encuentra el tema que nos ocupa, el autismo infantil. ¿A quién le puede extrañar que el epígrafe donde se ubica este cuadro pase a llamarse Trastornos del Desarrollo Neurológico? La idea de un sustrato neuronal patológico causante de estos y otros problemas infantiles como el TDAH no es precisamente una novedad ni es mayoritariamente rechazada, a pesar de que las voces críticas son cada vez más numerosas y contundentes (9-11). No obstante, aun siendo de crucial importancia la explicitación de esta hipótesis etiológica, no es el foco de nuestro artículo.

En la forma en que el autismo se engrana en la nueva clasificación, nos encontramos con otro cambio conceptual que, en realidad, lleva años admitido. Nos referimos a la fusión del Trastorno Autista, del Trastorno Desintegrativo Infantil y del Síndrome de Asperger -las categorías del DSM-IV-TR (12)-en el Trastorno del Espectro del Autismo, trasunto del popular Síndrome del Espectro Autista. El camino que va del autismo como un cuadro severo y con una bajísima incidencia a la idea de un "espectro" con un vago punto de corte que separaría lo normal de lo patológico, se empezó a recorrer al final de la década de los 70 del siglo pasado $(13,14)$. Por aquel entonces quedaba desterrado del lenguaje, paradójicamente, el propio autismo, pues en los artículos citados se consideraba poco afortunado el concepto de autismo infantil (13), para luego proponer englobar el espectro autista bajo el nombre de Síndrome de Asperger (14), el mismo que hoy desaparece. Para entender el rocambolesco vaivén es necesario hacer un poco de historia.

Agua y aceite: Alteraciones autistas del contacto afectivo de Kanner vs. Psicopatía autista en la infancia de Asperger

Resulta revelador cómo justifica Wing en su Asperger's Syndrome: A Clinical Account (14) la utilización del constructo "Síndrome de Asperger" como nueva categoría que daba nombre a una agrupación o cluster de niños formulada mediante análisis factorial (13) y cuyas características poco tenían que ver con los descritos por Asperger (15). Para la autora, este cluster, estos niños "podían quedar agrupados bajo la etiqueta amplia, pero insatisfactoria de psicosis infantil temprana". Para ella era más "satisfactorio" utilizar un nombre, el de Síndrome de Asperger, al que despojaba de su contenido original. ¿Qué tenía de satisfactoria una agrupación estadística ence- 
ORIGINALES Y REVISIONES

rrada en un nombre poco adecuado? Aun sin tener en cuenta motivaciones personales -la doctora Wing comenzó a estudiar el autismo a raíz del diagnóstico de su propia hija (16)- el apartar a los niños, aunque solo fuera en el lenguaje, de la psicosis o la esquizofrenia está en la base de la creación del concepto de autismo como enfermedad infantil de base neurológica.

Ya son más de 100 lo años que han pasado desde que Bleuler (17) mencionó el autismo como una de las características de los cuadros esquizofrénicos, pero en ningún caso como una cuestión central (18). Decía Bleuler: "Los esquizofrénicos se han apartado tanto como les ha sido posible de cualquier contacto con el mundo. Este alejamiento de la realidad con el consiguiente predominio de su mundo interno, es a lo que llamamos autismo".

Sin ser nuestra intención discutir acerca de la validez del diagnóstico de esquizofrenia o las diferentes formas de ser conceptualizado este cuadro, queremos recalcar la idea de que el autismo, en su origen, era una pequeña parte de un cuadro psiquiátrico grave, muy complejo y de baja prevalencia.

Con la llegada del trabajo de Kanner (19), el autismo pasa a ocupar el centro de los focos en los cuadros de Dementia Praecox en la infancia. Recuperamos aquí el término clásico popularizado por Kraepelin porque Kanner necesitaba algo para diferenciar su pretendido descubrimiento del resto de las psicosis infantiles más desorganizadas, que ya habían sido descritas incluso antes de la aparición del término "esquizofrenia" (20). A pesar del título de su trabajo, no es en el autismo donde Kanner sitúa la diferencia: "El signo patognomónico fundamental es la incapacidad para relacionarse en la forma habitual con personas y situaciones desde el comienzo de su vida ... no es una retirada, como en la esquizofrenia". (cursivas mías C.M.J.)

Como podemos ver, es el criterio temporal, el momento de aparición de la enfermedad lo que le sirve a Kanner para reclamar una diferencia respecto de la Dementia Infantilis de Heller (1908) o la Dementia Praecocissima Catatonica de De Sanctis (1908) y no la centralidad del autismo. En su famoso artículo -después de mencionar a Heller y De Sanctis-, Kanner indicaba que ambos cuadros necesitaban al menos dos años de desarrollo normal para su diagnóstico. Con la llegada del DSM-5, desaparece el criterio temporal que daba carta de naturaleza a las Alteraciones Autistas del Contacto Afectivo. Con la desaparición de este criterio, también lo hace la Dementia Infantilis, que había aguantado el tipo hasta hace poco bajo el nombre de Trastorno Desintegrativo Infantil o Síndrome de Heller. Como contrapunto, de Sanctis toma el relevo de la resistencia de los clásicos en la quizás inesperada especificación "con catatonía" que puede acompañar en el DSM-5 el diagnóstico del Trastorno del Espectro del Autismo. Más adelante intentaremos entender por qué.

¿Qué ha habido que hacer para que aquello que definía un cuadro y lo distinguía de otros -el criterio temporal- pueda ser eliminado no solo sin que desaparezca este cuadro, sino que, paradójicamente, haga desaparecer al resto? Como apuntába- 
mos arriba, la desaparición de la psicosis y de la esquizofrenia infantiles se encuentran, si no en la causa, sí en la consecuencia de esta maniobra. Las palabras de Donald Winnicott (21), pertenecientes a la reseña sobre un libro dedicado al autismo de Bernard Rimland (22), nos pueden servir para ilustrar la perplejidad que causa indagar en estas cuestiones: "Conocí la formulación de Kanner acerca del autismo luego de haber recogido una considerable experiencia en la psicosis de la niñez y nunca vi con claridad los motivos por los cuales debía separarse del tema total de la esquizofrenia en la infancia".

No era Winnicott el único que no lo veía claro. También en otras críticas a la concepción de Kanner figura la alusión a la "insatisfactoria" psicosis infantil como un concepto del que no tenía sentido deshacerse y el cuestionamiento del autismo como enfermedad neurológica congénita (23), algo que, por más que algunos lo pretendan ignorar, sigue lejos de estar demostrado (9).

Aun cuando Kanner mencionaba como patognomónica la retirada del mundo social, hemos visto que el autismo, en su origen, era una característica de un cuadro grave e incapacitante que iba mucho más allá de dicha retirada, y que otros autores lo encuadraban abiertamente en la esfera de la psicosis o la esquizofrenia infantiles. Será necesario que un trabajo olvidado durante 30 años aparezca en escena para que el autismo se desligue definitivamente de sus raíces psicóticas y pase a ser contemplado como una especie de rasgo de personalidad con extremos patológicos, pero del que todos somos portadores en alguna medida. Nos referimos al trabajo de Hans Asperger (15). A pesar de la confusión que ha originado la utilización de la obra del austriaco (24), Asperger dejaba claro de qué estaba hablando y de qué no: "La personalidad autista es claramente distinguible a pesar de las diferencias individuales. No son por lo tanto psicóticos, en cambio muestran un mayor o menor grado de psicopatía".

"Personalidad autista", "psicopatía" como equivalente clásico de los actuales "Trastornos de la personalidad", alusión franca a la ausencia de desintegración psicótica. Que con su "Psicopatía autista" Asperger estaba hablando de una cosa bien distinta de aquello que Kanner se esforzaba por distinguir de las esquizofrenias infantiles, queda bastante claro si se comparan los casos que ambos autores describen en sus artículos originales. La propia Lorna Wing (14) escribe así acerca de la agrupación estadística de niños que "descubrió" junto con Gould: "Muy pocos hacían recordar a la descripción dada por Asperger, algunos eran autistas de Kanner típicos. Otros podían ser incluidos de forma tentativa en síndromes descritos por autores como De Sanctis, Earl, Heller y Mahler, pero las definiciones dadas por estos autores no son lo suficientemente precisas para una adecuada clasificación. Todos podían ser agrupados en la categoría general, pero insatisfactoria, de psicosis infantil temprana".

Reubiquémonos: el párrafo anterior pertenece al artículo en el que se propone la expresión "Síndrome de Asperger" para agrupar a unos niños que, en su mayoría, 
coincidían con las descripciones dadas para niños autistas, psicóticos o esquizofrénicos, y "muy pocos" recordaban a los descritos por Asperger. La pregunta constante al realizar este trabajo ha sido ¿por qué? ¿Por qué todo tiene aparentemente tan poco sentido? Pero si tenemos en mente que la idea es esconder la triste, pero tozuda realidad-que la psicosis, con mayor o menor desintegración de la experiencia individual, también alcanza a los niños- podemos encontrar cierto sentido al afán de Wing. En efecto, si este cluster, si estos niños no eran psicóticos, ¿qué eran? El movimiento que realiza Wing para contestar es el siguiente:

$1^{\circ}$ Tenemos un cluster estadístico que agrupa a una serie de individuos. El contenido de los problemas que presentaban es lo que hoy conocemos como "Triada de Wing": "ausencia o dificultades en la interacción social, ausencia o dificultades en el uso del lenguaje, tanto verbal como no verbal, y ausencia o escasez de actividades verdaderamente flexibles e imaginativas, sustituidas por un estrecho rango de intereses repetitivos y estereotipados". La evaluación de las conductas se llevó a cabo mediante una entrevista estructurada a padres y cuidadores creada por las autoras para la evaluación de conductas en niños "retrasados y psicóticos" (25).

$2^{\circ}$ Puesto que los tres aspectos mencionados arriba se daban en diferente grado de severidad y asociados con distintos niveles de inteligencia, no procedía aplicarles el nombre de "autistas" que les podría corresponder porque esta etiqueta traía a la mente los casos más graves y asociados a inteligencia baja, pero dejaba fuera al resto.

$3^{\circ}$ Por motivos de "insatisfacción", quedaba descartado el recurso a seguir explorando el terreno de las psicosis infantiles y no convenía nombrarlas.

$4^{\circ}$ Puesto que Asperger había utilizado el término "autismo" en el título de su trabajo y describía problemas que tienen que ver en algún sentido con los que aparecían en la agrupación estadística, ¿por qué no llamar a este nuevo cuadro Síndrome de Asperger? Que el autor fuera totalmente desconocido en el mundo anglosajón ayudó (hemos leído que no hubo una traducción fiable de su obra al inglés hasta el trabajo de Uta Frith (15), pero es algo que no podemos valorar).

$5^{\circ}$ Poniéndose la venda antes de la herida, la autora reconoce que no hay más que una agrupación estadística de conductas problemáticas detrás de esta etiqueta, pero que eso puede ser muy conveniente por su utilidad: si tienen déficits en los mismos aspectos aunque en distinto grado, necesitarán intervenciones similares, aunque de distinta intensidad.

Y así quedaba desterrada por fin la psicosis infantil al tiempo que una teoría "ateórica" (mera estadística) proponía el concepto de déficit y la intervención compensatoria. La recientemente fallecida Lorna Wing (26) llegó a tiempo de disfrutar el refrendo de su pirueta psicopatológica al ver recogida en el DSM-5 su particular visión del autismo. Además, en este punto, después de hablar tantos años del espectro autista, y bien instalada esta idea en la mente de clínicos y legos, ya no era necesario utilizar a Asperger, por lo que el criterio que aludía en el DSM-IV-TR a la afectación 
o no del lenguaje desaparece y con él la posibilidad del diagnóstico de Síndrome de Asperger. El Síndrome del Espectro del Autismo ha conseguido barrer del mapa 100 años de psicopatología infantil.

Ahora se cierra el círculo conceptual y se entiende mejor la función instrumental que cumplió el trabajo de Asperger en todo esto. A la pregunta anterior, si estos niños no eran psicóticos, ¿qué eran?, ahora podríamos contestar algo así: son individuos, de cualquier edad, que, debido a una alteración neuronal, ocupan un lugar que supera el umbral de lo patológico en un rasgo de personalidad relacionado con la necesidad de tener y la habilidad para proporcionarse y mantener contactos sociales significativos, que tienden a ocuparse en intereses que no necesitan compartir y que pueden llegar a ser muy restringidos. Puesto que el rasgo principal está descrito en positivo, en función de tener (capacidad, necesidad, habilidades sociales), lo patológico es una ausencia, un déficit (no tener o tener poca capacidad, necesidad o habilidades sociales).

\section{La cuestión deficitaria y el espejo del retraso mental}

El capítulo del DSM-5 Trastornos del Desarrollo Neurológico engloba, entre otros, las discapacidades intelectuales, los trastornos de la comunicación, el espectro autista y el TDAH. En todos subyace el concepto de déficit (intelectual, de atención, en la comunicación) del que, como ya apuntaba Wing en el caso del autismo, se desprende una intervención basada en "management and education" (14). Con este tipo de intervenciones se "manejan" las peculiaridades a las que dan lugar los defícit y se "educa" a los niños para contrarrestarlos en la medida de lo posible. En el mismo trabajo, la doctora Wing dejaba la puerta abierta a una intervención clínica en caso de "problemas psiquiátricos superpuestos", pero la educación y la modificación de conducta eran las intervenciones propias en el autismo.

El modelo que se encuentra detrás es el del retraso mental, el de la discapacidad intelectual. En la nueva clasificación americana, el nivel de apoyo necesario para cada niño es una de las especificaciones del diagnóstico del Síndrome del espectro del autismo, al estilo de cómo era especificado el retraso mental por la Asociación Americana del Retraso Mental, hoy Asociación Americana por la Discapacidad Intelectual y del Desarrollo (27). Este criterio funcional para describir la discapacidad intelectual también ha sido trasladado al DSM-5. El nivel de gravedad lo determina el mayor o menor nivel de autonomía que se espera en cada caso, un enfoque que, en el retraso mental, impide que el rendimiento intelectual evaluado de forma estandarizada se convierta en una losa que impida ver más allá. Además, al ser el criterio de adaptación menos estable que el nivel de inteligencia, de ahí se desprende que, con 
un método de aprendizaje adaptado y un apoyo consistente, la persona con discapacidad intelectual puede alcanzar niveles de autonomía y satisfacción vital más altos y modificables a lo largo del tiempo. Este modelo es el que se ha trasladado al autismo.

El interés por desentrañar el recorrido que iba desde de la dementia praecocissima de principios del siglo XX, pasando por la psicosis y la esquizofrenia infantiles, para llegar al actual Síndrome del Espectro Autista no era un mero ejercicio escolástico: las intervenciones que se derivan de uno u otro enfoque (déficit cognitivo frente a psicosis) son diametralmente opuestas y, aunque consideramos que no son incompatibles -más bien nos parecen complementarias-, la negación en el lenguaje del término psicosis infantil hace que se destierren las intervenciones clínicas que abordan directamente el autismo desde un punto de vista psicopatológico, y dejan en el terreno de la psiquiatría o la psicoterapia las condiciones clínicas "superpuestas" (ansiedad, depresión, problemas de conducta, de regulación emocional, etc.).

\section{De lo deficitario a la defensa autista}

La experiencia clínica con niños autistas fue el principal motor de este artículo. Cuando, al salir a recibir a un preadolescente de 12 años a la puerta del despacho, éste te saluda agitando la mano y con un sonoro "ihola!", como si estuvieras a una docena de metros y tratara de hacerse ver, uno tiene la sensación de que algo no cuadra. Se trata de un saludo estereotipado, que nos hace pensar en un adiestramiento de años sobre conductas adecuadas e inadecuadas para relacionarse. Al revisar la historia, aparece un extenso informe de una universidad española, en el que se relata el procedimiento basado en la repetición mediante el que se implantan este tipo de conductas. Se alude a los avances conseguidos en los contactos sociales.

Bien, piensa uno, si con esto mejoramos las posibilidades de relacionarse de un niño, qué bien. Pero cuando uno habla con el chico, deja de tener las cosas tan claras. La descripción que hace de sus relaciones sociales como una lucha terrible que, además, parece ocurrir en su interior en lugar de que ser algo que le afecta, pero que está fuera, podría explicar la opresiva sensación que queda tras una hora de consulta. El tormento al que su propia elaboración del contacto con los demás le parecía conducir, no era mayor que el asombro que producía la descarnada descripción de sus distintos "yoes", enzarzados, además, en otra pelea interna que le escindía. La pregunta que queda en el aire es ¿sobre qué experiencia del propio yo, de sus límites y de lo que son los otros estamos implantando los aprendizajes que preconiza la visión deficitaria que se impone sobre estos niños?

Nos encontramos ahora en la consulta con un niño de 5 años. La repetición incansable de lo que parece una escena de un videojuego de plataformas que termina 
con el protagonista despeñándose - dos dedos simulando un personaje que corre, salta y termina cayendo al vacío-, apenas deja hueco para hacer otra cosa que marcar una cruz en "comportamiento repetitivo o estereotipado". Pero el psicólogo insiste, hasta que parece atinar con la pregunta. Un escalofrío recorre al profesional al escuchar la respuesta. El niño, que alterna la conducta anterior con la reproducción de diálogos de unos dibujos de su superhéroe favorito, se detiene al escuchar la siguiente pregunta: "Y cuándo no hay películas, ¿qué hay?" Mira al psicólogo y contesta: "Ay, qué dolor", para continuar acto seguido con su cantinela. Decía Winnicott (28), hablando de las intervenciones en psicosis infantiles graves: "Si tenemos éxito, posibilitaremos que el paciente abandone la invulnerabilidad y se convierta en una persona que sufre".

Una forma de entender el autismo diferente de lo deficitario lo sitúa como algo positivo, esto es, no como una carencia, sino como una construcción, un muro de protección. El problema es que el muro es de tal modo inexpugnable que parece impedir la existencia más allá de lo que constituye la propia defensa masiva. Pero, "si tenemos éxito", ocurren cosas. Terminado el tiempo de la consulta con el paciente anterior, nos dirigimos hacia la puerta tras la que se encuentran sus padres. El niño, ya fuera de la consulta, se gira un momento y alza sus manos hacia mí -lo que interpreto como un gesto de afecto- y le tiendo los míos, sin entender aún del todo. El niño agarra mis manos y les da la vuelta para situarlas formando un cuenco ante la mirada expectante de los padres, que presencian la escena. Acto seguido, lleva su mano derecha a la cabeza, simula que toma cosas de allí y las deposita en el improvisado recipiente. De nuevo con el corazón encogido, disimulo mientras le digo a los padres, quitándole importancia: "¿Que qué hace? Es lo que hacemos en la consulta, ¿no?, dejar aquí algunas cosas que nos rondan por la cabeza”.

Recuperar el enfoque clínico sin menospreciar las intervenciones educativas y de modificación de conducta

Parece razonable pensar que no sea especialmente ducho en habilidades sociales alguien para quien salir al mundo es un tormento. Con un enfoque que niega ese sufrimiento -o lo considera una consecuencia de la falta de habilidades- nos vemos impelidos a llevar a cabo intervenciones estereotipadas y descarnadas basadas en principios básicos de aprendizaje y modificación de conducta, evitando enfrentarnos a esa angustia terrible y sin nombre para quien la padece que los de este lado llamamos -seguramente de forma insatisfactoria- angustia psicótica. Solo la humanidad de los profesionales que tienen la capacidad para trabajar con estos niños compensa el origen deshumanizante de las teorías que sustentan estas intervenciones: es un 
cerebro que no funciona bien, un hardware al que no se le instaló un programa que debería venir de serie.

Estos puntos de vista organicistas o cognitivos, siendo por completo descarnados, tienen mejor prensa de la que seguramente merecen gracias a la asociación que todavía hoy persiste entre el autismo y las hipótesis etiológicas relacionadas con la idea de madre nevera o madre rechazante (29). Ya estaba en Kanner (19) una alusión a la ausencia de padres "verdaderamente afectuosos", madres de alto nivel sociocultural y profesiones liberales en padres de ambos sexos, varios de los cuales eran psiquiatras, entre los casos que él describe. Esta leyenda negra sobre los padres de los niños autistas hace mucho daño a la hora de que podamos aceptar el sufrimiento intrínseco a su condición en estos niños y poder abordarla desde un punto de vista clínico, sin que para ello haya que abandonar el abordaje educativo; pero hay esperanza. Sin olvidar que pueden darse casos de padres verdaderamente incapaces, podemos encontrar otros padres con hijos autistas que abogan por un abordaje distinto de este problema (30), dando una lección a muchos profesionales que parecen querer defender a los padres del sufrimiento de sus hijos a costa de convertirlos en "pedagogos exigentes y entrenadores de nuestros hijos", y asumiendo que queda mucho por entender sobre lo que es el autismo antes de cubrirlo de un manto educativo y de control de la conducta.

\section{De Sanctis o la tozuda realidad}

Pensamos que la inclusión en el DSM-5 de la especificación "con catatonía" en el diagnóstico del espectro autista es un vestigio del pasado conceptual del autismo que se resiste a abandonar el escenario. Al pasar del autismo como un adjetivo en las psicosis infantiles, tal y como lo concebía Kanner, a convertirlo en un sustantivo, hoy es la catatonía la que se añade como adjetivo, como signo de máxima gravedad en ese Espectro Autista que, sin esa especificación, podría dejar fuera muchos de los casos que estaban en el origen de estas indagaciones. Es el peaje a pagar por el salto que ya hemos descrito: de un cuadro psiquiátrico grave por definición y de baja prevalencia, a algo parecido a un rasgo de personalidad que puede llegar a ser problemático, que es lo que se esconde tras el concepto de espectro, un continuo en el que ubicar a las personas. Creemos que la constante revisión al alza de los datos epidemiológicos de estos diagnósticos tiene que ver con esta forma tan particular de entender los trastornos.

En un sentido similar, el modelo de la discapacidad es muy atractivo porque ofrece baremos del déficit de los que se desprende el volumen de la ayuda necesaria. Medir y cuantificar, seguros médicos y apoyos escolares, la lógica del mercado se instala en la descripción clínica, aunque se pueda tratar en muchos casos de recursos públicos. Pensamos que en este enfoque cuantitativo se encuentra uno de los proble- 
mas de la lógica del espectro en psicopatología. Heredero del enfoque de rasgos en psicología de la personalidad, todos podemos ser ubicados en un punto u otro de un espectro psicopatológico. O, visto desde los rasgos, todos podemos ser ubicados en un punto con respecto a un rasgo de personalidad, por ejemplo, sociabilidad, y pasarán a ser considerados patológicos los extremos del continúo. Una consecuencia es que, al incluirnos todos en todos los "espectros" desaparecen las categorías en psicopatología. Todos somos susceptibles de puntuar esto o aquello en tal o cual espectro. Seguramente en esta herramienta se pueda ver uno de los mecanismos que están detrás de las epidemias psiquiátricas que asolan occidente. Al desaparecer las categorías clínicas, los puntos de corte en los espectros establecen una difícil decisión entre la patología leve y la normalidad, al tiempo que los enfermos mentales graves dejan de ser dueños de su propio padecimiento, que es usurpado por la población general, ávida de consumir, aunque sean remedios psiquiátricos. Como vemos, los problemas que introduce la idea de espectro en psicopatología trasciende el asunto que estábamos abordando, pero, como suele ocurrir, en el caso de los niños las repercusiones son más dolorosas. Por un lado, caerán en el espectro autista en su forma leve niños tímidos, callados o con intereses particulares, que no deberían entrar en contacto con el circuito psiquiátrico, al mismo tiempo que los casos más graves se ven privados de una identidad que abarque todas las particularidades de su experiencia, limitando el reconocimiento de su probable sufrimiento a lo definido por el espectro de turno.

\section{Conclusión}

Como decíamos al principio, tratábamos de poner de manifiesto de qué forma la teoría implícita tras un diagnóstico limita la intervención a aquello que dicha teoría era capaz de nombrar. Creemos que no andamos lejos de poder afirmar que esto ocurre en gran medida en lo que concierne al autismo, pues la carga teórica del diagnóstico impide que pasen a un lugar central aspectos que eran esenciales cuando se hablaba de psicosis infantil. Para nosotros, la cuestión no pasa tanto por decidir qué forma de entender estos casos es la mejor, sino por poder evitar que una concepción concreta del mismo, la del déficit cognitivo, acabe por negar otros componentes del cuadro, si no más importantes en su descripción clínica, seguramente más cercanos a la experiencia de las personas que lo padecen. Con los niños autistas, no podemos entregarnos al modelo de la discapacidad de una forma completa, como ocurre en algunas ocasiones, y renunciar al estudio de y a la intervención en estos cuadros desde otros puntos de vista. 
ORIGINALES Y REVISIONES

\section{BIBLIOGRAFÍA}

(1) American Psychiatric Association. Diagnostic and statistical manual of mental disorders (5th ed.). Arlington, VA: American Psychiatric Publishing; 2013.

(2) González Menéndez AM, Williams G, Pérez-González LA. Guía de tratamientos psicológicos eficaces para el autismo. En Pérez Álvarez M, Fernández Hermida JR, Fernández Rodríguez C, Amigo Vázquez I (Eds.). Guía de tratamientos psicológicos eficaces III: Infancia y adolescencia (pp. 17-56). Madrid: Pirámide, 2003.

(3) American Psychiatric Association. Diagnostic and statistical manual of mental disorders (3rd ed.). Washintgton, DC: APA, 1980.

(4) Frances A. Saving Normal: An Insider's Revolt Against Out-of-Control Psychiatric Diagnosis, DSM-5, Big Pharma, and the Medicalization of Ordinary Life. New York: HarperCollins, 2013.

(5) McGorry PD, Killackey E, Yung A. Early intervention in psychosis: concepts, evidence and future directions. World Psychiatry. 2008;7(3):148-156.

(6) Corcoran C, Malespina D, Hercher L. Prodromal interventions for schizophrenia vulnerability: the risks of being "at risk". Schizophr Res. 2005;73(2-3):173-184.

(7) Warner R. Problems with early and very early intervention in psychosis. Br J Psychiatry. 2005:187(Suppl 48):s104-s107.

(8) Mercier A, Auger-Aubin I, Lebeau JP, Schuers M, Boulet P, Van Royen P, et al. Why do general practitioners prescribe antidepressants to their patients? A pilot study. Biopsychosoc Med. 2014;8:17.

(9) Timimi S, Gardner N, McCabe B. The Myth of Autism. Medicalisin Men's and Boy's Social and Emotional Competence. Humpshire: Palgrave Macmillan, 2011.

(10) Gnaulati E. Back to Normal. Why ordinary Childhood Behavior is Mistaken for ADHD, Bipolar Disorder and ASD. Boston: Beacon Press, 2013.

(11) García de Vinuesa F, González Pardo H, Pérez Álvarez M. Volviendo a la normalidad. La invención del TDAH y del trastorno bipolar infantil. Madrid: Alianza editorial, 2014.

(12) American Psychiatric Association. Diagnostic and statistical manual of mental disorders (4th ed. rev.). Washintgton, DC: APA, 2000.

(13) Wing L, Gould J. Severe impairments of social interaction and associated abnormalities in children: epidemiology and classification. J Autism Dev Disord. 1979;9(1):11-29.

(14) Wing L. Asperger's Syndrome: A Clinical Account. Psychol Med. 1981;11:115-129.

(15) Asperger H. Autistic Psychopathy in Childhood. 1944. En Frith U. Autism and Asperger Symdrome. Cambridge: Cambridge University Press, 1991.

(16) Rhodes G. Autism: a mother's labour of love. The Guardian [edición digital] may-242011 [consultado oct-22-2014]. Disponible en http://www.theguardian.com/lifeandstyle/2011/may/24/autistic-spectrum-disorder-lorna-wing

(17) Bleuler E. Dementia Praecox oder Gruppe der Schizophrenien. Leipzig: Deuticke, 1911.

(18) Moskowitz A, Heim G. Eugen Bleuler's Dementia Praecox or the Group of Schizophrenias (1911): A Centenary Appreciation and Reconsideration. Schizophr Bull. 2011;37(3):471-479.

(19) Kanner L. Autistic Disturbances of Affective Contact. Nerv Child. 1943;2:217-250.

(20) Rojas DR, Outes M, Cositore A. Sante de Sanctis y la Demencia Precocísima. Alcmeón. 1996;5(3):277-288. 
ORIGINALES Y REVISIONES

(21) Winnicott DW. Study of Autism. Br Med J. 1966;2(5514):634.

(22) Creak M. Psychosis in Childhood. J Ment Sci. 1951;97:545-554.

(23) Rimland B. Infantile autism. New York: Appleton-Century-Crofts, 1964.

(24) Schopler E. Premature Popularization of Asperger Syndrome. En Schopler E, Mesibov GB, Kunce LJ. Asperger Syndrome or High-Functioning Autism? New York: Plenum Press, 1998.

(25) Wing L, Gould J. Systematic recording of behaviors and skills of retarded and psychotic children. J Autism Child Schizophr. 1978;8:79-97.

(26) Gould J. Lorna Wing Obituary. The Guardian [edición digital] jun-22-2014 [consultado oct-22-2014]. Disponible en:

http://www.theguardian.com/society/2014/jun/22/lorna-wing

(27) Schalock RL, Borthwick-Duffy SA, Bradley VJ, Buntinx WHE, Coulter DL, Ellis MC, et al. Intellectual Disability: Definition, classification, and systems of supports (11 ${ }^{\text {th }}$ ed.). Washington: American Association on Intellectual and Developmental Disabilities, 2009

(28) Winnicott DW. La esquizofrenia infantil en términos de adaptación. 1967. En Pérez A, Dimant F (edit.) Psicosis Infantil. Buenos Aires: Nueva Visión, 1980.

(29) Bettelheim B. The empty fortress. New York: The Free Press, 1967.

(30) Manifiesto del 2 de abril de la asocación TEAdir. Disponible en http://associacioteadir. org/wp-content/uploads/2014/03/manifest.pdf 